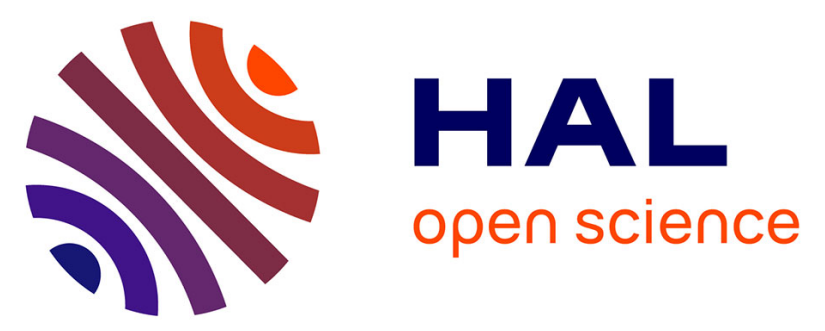

\title{
Digitalisierung und ihre Einflüsse auf den Umgang mit alten wie neuen 'Briefen' in deutscher wie internationaler Perspektive
}

Anne Baillot

\section{- To cite this version:}

Anne Baillot. Digitalisierung und ihre Einflüsse auf den Umgang mit alten wie neuen 'Briefen' in deutscher wie internationaler Perspektive. De Gruyter. Handbuch Brief. Von der Frühen Neuzeit bis zur Gegenwart. Band 1: Interdisziplinarität - Systematische Perspektiven - Briefgenres, p. 387398, 2020, Band 1: Interdisziplinarität - Systematische Perspektiven - Briefgenres, 978-3-11-037510-7. hal-02543927

\section{HAL Id: hal-02543927 \\ https://hal.science/hal-02543927}

Submitted on 16 Apr 2020

HAL is a multi-disciplinary open access archive for the deposit and dissemination of scientific research documents, whether they are published or not. The documents may come from teaching and research institutions in France or abroad, or from public or private research centers.
L'archive ouverte pluridisciplinaire HAL, est destinée au dépôt et à la diffusion de documents scientifiques de niveau recherche, publiés ou non, émanant des établissements d'enseignement et de recherche français ou étrangers, des laboratoires publics ou privés. 


\section{Digitalisierung und ihre Einflüsse auf den Umgang mit alten wie neuen „Briefen“ in deutscher wie internationaler Perspektive Anne Baillot (Le Mans Université)}

\section{Begriffsbestimmung}

Unter Digitalisierung im weitesten Sinne fallen neben der eigentlichen Digitalisierung von physischen Gegenständen (Scans von zwei- oder dreidimensionalen Artefakten; Schreibman et al. 2004, IV.32.) eine Reihe anderer Verfahren und Informationswandlungen, die das so erstellte Digitalisat online auffindbar machen: die Erfassung von Metadaten, die manuelle, semi-automatische oder automatische Extraktion von Textinformationen (inkl. Annotationen) und deren Einbettung in einschlägige online-Sammlungen (Datenbanken, Repositorien, Editionen). Für forschungsrelevante Materialien hat die digitale Aufbereitung nach etablierten Standards zu erfolgen, welche die Bedingung für die Anerkennung im wissenschaftlichen Bereich ausmachen. Mit den vier o.g. Schritten sind Techniken verbunden, die sich teilweise noch in Entwicklung befinden.

Hochleistungsscanner ermöglichen es, empfindliche Materialien beim Scannen physisch unberührt zu lassen (etwa die Totes-Meer-Rollen; https://uknow.uky.edu/content/scroll-engedi-high-tech-recovery-mission) oder zerbrechliche Materialien einmal zu erfassen, um die Rekonstruktion der Bruchstücke digital nachzuvollziehen, um dabei die materielle Quelle maximal zu schonen (wie bei den Elephantine Papyri: http://blog.smb.museum/papyri-vonelephantine-wie-aegyptologen-die-antike-decodieren/). Bei Massendigitalisierungen dagegen ist die niedrige Qualität der Scans für die Nutzung und weitere Bearbeitung oft hinderlich (vgl. die Analyse von Klaus Graf zur Scanqualität in der BSB Müchen: https://www.inetbib.de/listenarchiv/msg39553.html). Das Qualitätsspektrum ist breit gefächert. Grundsätzlich entwickelt sich die Scanqualität weiter, sodass es sich bei diesem Verfahren zwar um eine Aufbewahrungs- und Schonungsmaßnahme handelt, die aber nicht zwangsläufig mit einem einmaligen Bearbeiten verbunden ist. Gegebenenfalls kann (bzw. soll oder muss) ein solcher Gegenstand im Laufe der Zeit wiederholt gescannt werden, um etwa die Digitalisierungsqualität zu verbessern (zu Best Practices der Digitalisierung vgl. ETH Bibliothek 2016; Neudecker und Rehm 2016).

Der digitalen Erfassung des jeweiligen Gegenstandes muss eine Beschreibung bzw. zusätzliche Information an die Seite gestellt werden. Ohne diese Metadaten sind die erstellten Bilder, etwa von Briefhandschriften oder gedruckten Briefen, nicht auffindbar. Die Verbindung von Scans mit Metainformationen über den Gegenstand (sowie evtl. auch über den Scan selbst, seine Qualität, seine Herstellung oder den bzw. die Rechteinhaber_innen) ist die unerlässliche Bedingung für ihre Online-Auffindbarkeit und -Zuordnung. Die meisten Wissenschaftler_innen kennen Metadaten in der Regel nur aus Bibliotheks- oder Archivverzeichnissen. Vor dem Aufkommen digitaler Methoden wurden sie meist nicht als Teil der Forschungsarbeit betrachtet. Die Einbettung der unterschiedlichen Informationsebenen im Internet macht sie jedoch zu leistungsstarken digitalen Schnittstellen. Es gibt derzeit kein nationales oder internationales einheitliches Format für solche Metadaten (vgl. bspw das Europeana Data Model, http://pro.europeana.eu/files/Europeana Professional/Share your data/Technical require ments/EDM Documentation//EDM Definition v5.2.7 042016.pdf). Für Briefe ist insbesondere zu unterstreichen, dass gemeinsame Bestrebungen von Bibliotheken, Archiven und Forschung derzeit auf die Definition von für Briefe relevanten Grundinformationen und 
ihrer Kommunizierbarkeit innerhalb von Einrichtungen abzielen: Absender, Adressat(e), Absendedatum, Absendeort, Umfang bilden die archivarische Visitenkarte eines Briefes aus und lassen sich sowohl in archivarischen Formaten (EAD, METS) als auch in wissenschaftlichen Formaten (XML/TEI) erfassen. Die Möglichkeit, die Kommunikation zwischen den Metadatenformaten zu ermöglichen, ist ein anerkanntes Desiderat und wird sowohl von den einzelnen Institutionen als auch von den Forschungsinfrastrukturen (DARIAH Cultural Heritage Data Reuse Charter) bearbeitet.

Bei gedruckten und handschriftlichen Briefen stellt sich nach dem Scannen und dem Versehen mit Metadaten die Frage nach der Extraktion von Text in ein maschinenlesbares Format, das die weitere digitale Bearbeitung des Inhalts möglich machen wird. Automatische Zeichenerkennung (OCR) ist nach wie vor keine $100 \%$ zuverlässige Technik, sondern verlangt Kontrolle der Ergebnisse und Nacharbeit. Die erreichten Ergebnisse sind bei typographisch einheitlichen und sauberen Texten von weitgehend höherer Qualität als bei Schriftmischungen, Spaltenaufteilungen und welligem Papier (das Projekt OCR-D strebt an, diese Schwächen aufzufangen: http://ocr-d.de/). Automatische Handschriftenerkennung erreicht trotz des verstärkten Einsatzes von Machine Learning-Methoden (Hidden Markov Models, Deep Learning) noch keine Ergebnisse, die bei der Einbindung in die digitale Verarbeitung im Wesentlichen manuelle Arbeit signifikant ersparen würde. Nennenswert ist in diesem Zusammenhang das neuerdings entwickelte Tool Transkribus (https://transkribus.eu/), das einen Schwerpunkt in Sütterlin und früheren deutschen Schreibarten hat. Transkribus kann immer bessere Ergebnisse vorlegen, ist jedoch noch weit davon entfernt zu sein, manuelle Handschriftentranskription zu ersetzen (TranskribusNutzerberichte: http://www.etrap.eu/wp-content/uploads/2016/11/TrAIN-

Transkribus User Report-2016.pdf; http://read.transkribus.eu/wpcontent/uploads/2017/01/Report transkribus.pdf).

Das Zeichenerkennungsverfahren konzentriert sich auf die Zusammenstellung des im jeweiligen Dokument abzulesenden Fließtextes. Aber bei der digitalen Aufbereitung von Informationsextraktion kann man noch einen Schritt weitergehen. In diesem Fall geht es nicht ausschließlich um die (semi)automatische Generierung einer Transkription des Fließtextes des Briefes, denn nicht nur Text kann automatisch extrahiert oder manuell gesucht und markiert (,ausgezeichnet“) werden, sondern auch im Text enthaltene Informationen. Diese Technik lässt sich auf alle Textarten anwenden und die Instanzen je nach Bedarf bestimmen. Bei für Briefe relevanten Informationen sind Methoden am besten entwickelt, die es möglich machen, Entitäten (Personen, Orte, Werke) oder linguistische Informationen zum syntaktischen Textaufbau des Textes zu gewinnen. Zu diesen Zwecken können Verfahren verwendet werden: für NER (Named Entities Recognition; bspw mit GROBID, https://grobid.readthedocs.io/en/latest/), für Lemmatisierung und für Part of Speech-Tagging (etwa mit dem TreeTagger: Schmid 1994, Schmid 1995). Die Ergebnisse dieser diversen Erkennungsverfahren können im Nachgang für die Strukturierung der algorithmischen Auswertung der betroffenen Texte verwendet werden (bspw. Schöch 2016), für ihre Verbindung mit anderen online-Ressourcen (bspw. GND-BEACON, https://de.wikipedia.org/wiki/Wikipedia:BEACON) oder aber für die Visualisierung bestimmter (Teil)Informationen (bspw. mit Voyant: https://voyant-tools.org/).

Die Einbettung digitaler Informationen über Briefgegenstände in digitalen Sammlungen kann auf mehreren Ebenen und im Rahmen von unterschiedlichen institutionellen 
Zusammenhängen erfolgen. Denkbar ist beispielsweise bei einem Brief ein institutionelles Zusammenspiel, bei dem die Metadaten in einer übergeordneten Datenbank wie Kalliope (http://kalliope-verbund.info/de/index.html) oder Archiv-D (https://www.archivportald.de/) verfügbar gemacht werden; ein Scan auf der Webseite der bestandserhaltenden Institution (bspw. digitalisierte Sammlungen der Staatsbibliothek zu Berlin-PK:

http://digital.staatsbibliothek-berlin.de/), eine Transkription in einer wissenschaftlichen Edition und eine Visualisierung der Briefdaten in einem Portal, das die Erstellung von Netzwerkvisualisierungen ermöglicht (bspw. Nodegoat: https://nodegoat.net/; oder palladio: http://hdlab.stanford.edu/palladio/). Damit aber diese Instanzen, die alle von demselben Artefakt oder einer Repräsentation desselben ausgehen, mit einander verbunden sein und auf einander verweisen können, müssen die einschlägigen Informationen in einem Format erfasst werden, das die Kommunikation zwischen diesen Instanzen zulässt. Aus diesem Grunde ist ein Scan im pdf-Format eine digital gesehen äußerst informationsarme Ressource, ein digitales Waisenkind.

Der etablierte Standard, der es am ehesten möglich macht, dass Briefdaten in Verbindung mit einander gesetzt werden können, sind die von der Text Encoding Initiative etablierten Richtlinien (TEI-P5, TEI Consortium 2013). Diese dienen der Kodierung von Texten im Allgemeinen, mit einem besonderen Schwerpunkt auf geisteswissenschaftlich relevanten Texte. Neben für alle Textsorten gültige Auszeichnungsmöglichkeiten hat die TEl bestimmte Richtlinien für Briefe entwickelt, beispielswiese für den Brieftext die Elemente <opener> (http://www.tei-c.org/release/doc/tei-p5-doc/en/html/ref-opener.html), <closer> (http://www.tei-c.org/release/doc/tei-p5-doc/en/html/ref-closer.html) und <postscript> (http://www.tei-c.org/release/doc/tei-p5-doc/en/html/ref-postscript.html) um Briefkopf und -ende, die beim Briefeschreiben feste Bestandteile des üblichen modus scribendi sind, sowie Post-Skripta, festzuhalten. Im Fall von Briefhandschriften kann ebenfalls das TEIModul „Critical Apparatus“ für die Erfassung von textgenetischen Phänomenen herangezogen werden (http://www.tei-c.org/release/doc/tei-p5-doc/en/html/TC.html; auch Burghart 2017).

Für die Vernetzung von Briefdaten jedoch ist vor allem das Element <correspDesc $>$ von zentraler Bedeutung, da dieses auf Metadatenebene angesiedelt ist und als Plattform für den Austausch von Briefdaten fungieren kann (http://www.tei-c.org/release/doc/tei-p5doc/de/html/ref-correspDesc.html sowie Stadler et al. 2016). <correspDesc> erfasst Briefmetadaten auf der Ebene der Kommunikationshandlung (Adressat, Absender, Datum, Ort, Einbettung in eine Gesamtkorrespondenz), während die Beschreibung der handschriftlichen Spezifika (Materialität) innerhalb der Kategorie <fileDesc $>$ bzw. $<m s D e s c>$ erfolgt. Die auf der Grundlage von correspDesc entwickelte Plattform correspSearch ermöglicht die Suche durch Briefkorpora anhand des Metadatenaustauschformats CMI (http://correspsearch.net/index.xql?id=participate cmi-format\&l=de ).

Im Rahmen von Linked Open Data-Ansätzen

(https://www.w3.org/standards/semanticweb/data) wird auf die semantische Auszeichnung von Briefen verstärkt Wert gelegt. Die Texte werden in der Regel nicht nach den Richtlinien der TEl erfasst, sondern anderweitig annotiert. Aus RDF-Triples

(https://www.w3.org/TR/rdf11-concepts/) lassen sich Informationen extrahieren, die beispielsweise für die Visualisierung oder sonstige semantische Analyse der Briefinhalte einschlägig sind. Bei dieser Form der Kodierung ist jedoch die Verknüpfung mit anderen Ressourcen aufgrund der variierenden Ontologien und Vokabularien nicht einfach zu 
bewerkstelligen. In der Regel handelt es sich um Insellösungen, bei denen jede Ressource für sich im Netz steht, ohne unmittelbar mit anderen verknüpfbar zu sein.

\section{Theoretischer Unterbau}

Das mit digitalen Briefeditionen, -repositorien, -metadatenverzeichnissen und sonstigen datenbanken zusammenhängende Verlinkungspotential beantwortet ein lange zuvor bereits identifiziertes Forschungsdesiderat der Briefforschung. Briefe sind dadurch charakterisiert, dass sie nicht für sich bestehen, sondern auf externes Material, Ereignisse oder Personen verweisen. Ihre dialogische Struktur bettet Briefe in einen weitergehenden Informationszusammenhang ein. So steht die Verbindung jeder Briefinstanz mit den kontextuell relevanten Quellen und Informationen im Mittelpunkt der Untersuchung von und Arbeit mit Brieftexten. Digitale Methoden bieten mit Blick auf diese Fragestellung die Möglichkeit, sich von in der Printwelt einschränkenden, editorischen Herausforderungen bei der Präsentation von Briefmaterial (alle an-Briefe nach einander, dann alle von-Briefe, oder im Dialog; zwischen zwei Protagonisten oder alle Briefpartner_innen einschließend etc.) loszulösen und die Dialogizität zwischen Briefen, Korrespondenzpartner_innen und Texten mehrschichtig zu erfassen und wiederzugeben. Ein- und dieselbe Kodierung in TEI-P5 lässt bei entsprechender Bearbeitung unterschiedliche Webanzeigen zu, sodass man nicht mehr auf eine einzige editorische Aufbereitungsform angewiesen ist, sondern aufgrund derselben Erfassungsgrundlage die unterschiedlichen Aspekte etwa der Dialogizität wiedergeben kann (Baillot und Busch 2014).

Dies setzt allerdings voraus, die technischen Optionen, die auf die jeweilige Fragestellung zugeschnitten werden können, auszuloten und gezielt einzusetzen. Ebenfalls setzt es eine Zusammenarbeit zwischen Online-Ressourcen voraus, die es in der Printwelt nicht gibt, um die notwendige Verlinkung zwischen den Briefinformationen zu gewährleisten. Der wissenschaftliche Erkenntnisgewinn der Verknüpfung von Briefmaterial führt zur Institutionalisierung von Kooperationen und epistemologisch zu einer größeren Anpassung von den Anforderungen des Materials und denjenigen ihrer digitalen Bearbeitung und Präsentation: Was konzeptionell dialogisch ist, wird auch dialogisch umgesetzt.

Während die digitale Erfassung von historisch belegbaren, briefbezogenen Informationen im eben geschilderten Sinne zu einer neuartigen Potenzierung von Briefmaterial beiträgt und die Briefforschung neubelebt, ist es nach wie vor ein Desiderat digitaler Methoden, literarische Phänomene gebührend zu erfassen und in diesem Bereich eine Grundlage für Editionen bzw. Auswertungen zu schaffen. Die Literatur- und Kulturgeschichte hat sich eingehend damit befasst, dass es sich bei Briefinformationen nicht um die Schilderung objektiver Fakten handelt, sondern um die subjektive Inszenierung, ja Literarisierung eines Briefschreiber-Ichs im Kontext der jeweiligen betroffenen Korrespondenzen (Busch 2016). Die Erfassung von dem Briefhabitus geschuldeten Verzerrungsphänomenen (PseudoStreichungen, unterschiedliche Informationswiedergabe je nach Korrespondenzpartner_in ...) lässt sich digital nicht leicht erfassen und auch nicht leicht repräsentieren, sondern verlangt in der Regel einen dem Printhabitus ähnlichen Umweg über die Kommentarfunktion. Die Entwicklung von Auszeichnungsschemata, die auf literaturwissenschaftliche Fragestellungen zugeschnitten wären, ist ein wichtiges Forschungsdesiderat des beginnenden 21. Jahrhunderts (Ansätze bislang ausschließlich im Bereich der Auszeichnung von Zeit; Gius und Jacke 2016). In diesem Kontext wäre die Erfassung und Untersuchung von relevanten literarischen Inszenierungsphänomenen in Briefen ein ertragreicher Anwendungsfall. 
Briefanalyse entwickelt sich in der Regel in einem Balanceakt zwischen Fallbeispiel und allgemeinen Strukturanalysen. Der Briefhabitus eines Briefschreibers lässt sich nur im Kontext des Briefhabitus seines Umfeldes korrekt interpretieren; zeitgleich ist es die Summe der beobachtbaren Briefhabitus, die diesen Kontext ausmacht (Busch 2013). Bei der Informationsmenge, über die wir heute verfügen, ist es menschlich nicht möglich, alle für einen bestimmten Kontext relevanten Informationen sowohl im Detail als auch strukturell zu beherrschen. Digitale Methoden erleichtern die Pendelbewegung zwischen Mikro- und Makroanalyse in vielerlei Hinsichten. Sowohl in Worten der epistolaren Netzwerkbildung als auch mit Blick auf die Schreibtechniken lassen sich digital erfasste Briefkorpora miteinander auf einer Ebene vergleichen, die der Forscher/die Forscherin allein nicht bewerkstelligen kann. Eine wissenschaftlich zuverlässige Erfassung des Briefmaterials lässt darüber hinaus die Möglichkeit zu, von der Makro-Ebene bei Bedarf in die Mikro-Ebene einzutauchen, $d . h$. mit Methoden des Distant Reading relevante Textstellen zu identifizieren, an denen Close Reading sich als am ertragreichsten abzeichnet.

Bemüht man sich um ein einschließendes Verständnis des Begriffs „Brief“ (Bohnenkamp und Wiethölter 2008), fallen unter diesen Begriff ebenfalls originär digitale Kommunikationsformen wie E-Mails, ja gar, nimmt man den Briefbegriff sehr allgemein, andere Kurzformen wie Chat-Nachrichten oder Tweets. Bei diesen sogenannten born-digital Briefquellen stellt sich die Frage nach der Erfassung und Auswertung insofern anders, als sie oft im Rahmen von kommerziellen Angeboten entstehen, die eine dezidierte Informationskontrolle ausüben: Twitter liefert der Forschung immer nur einen Bruchteil der für eine Forschungsfrage relevanten Tweets, ohne deutlich zu machen, nach welchen Kriterien diese sortiert werden. Bei E-Mails stellen sich darüber hinaus Formatfragen: Nach einer gewissen Zeit wechseln Online-Formate bzw. sind nicht mehr miteinander kompatibel, sodass man ältere E-Mails beispielsweise nicht mehr digital lesen kann. So müsste ein forschungsrelevantes E-Mailkorpus zielgerichtet in ein Format überführt werden, das die Langzeitarchivierung ermöglicht. Da ein Großteil der Forschung, die derzeit solche Korpora in den Fokus nimmt, in den Sozialwissenschaften durchgeführt wird, werden sie in Textformaten durchgeführt, die den oben geschilderten Ansprüchen der Briefforschung nicht genügen (eine Ausnahme, von der Linguistik ausgehend und auf der TEl aufbauend, ist Beißwenger 2016).

Methodisch gesehen stellen born-digital briefähnliche Materialien die Briefforschung vor die Herausforderung der schieren Informationsmasse, die täglich produziert wird (Strobel 2012). Es lässt sich nicht deutlich erkennen, wie sich Briefforschung auf der Grundlage von rein elektronischen Formaten in historischer Perspektive entwickeln wird. Diese Sachlage stellt die Forschung vor den epistemologischen Bedarf, sich Konzepten der Archivierung und damit zusammenhängenden Sortierungs-, Filtrierungs- und Umformatierungsphänomenen zu widmen, was noch nicht in dem Ausmaß erfolgt ist, wie es die Arbeit mit diesen Materialien verlangen würde.

\section{Fallbeispiele}

Die digitale Aufbereitung von Briefmaterial ermöglicht fließende Übergänge zwischen Briefverzeichnissen, -editionen, -anthologien und -analysen. Innerhalb dieser Vielfalt an möglichen Darstellungen lässt sich hauptsächlich zwischen autorzentrierten und 
vernetzenden Korrespondenzaufbereitungen unterscheiden. Die meisten entstehen im Rahmen von wissenschaftlich bzw. archivarisch definierten und aufbereiteten Textgrundlagen. Eine Ausnahme bildet das Projekt „Letters of 1916“, das nicht nur die Transkription der Briefe, sondern auch die Zusammenstellung des Materials von den Beiträgen zahlreicher, auch unbekannten Teilnehmer_innen abhängig macht, welche privates Archivmaterial zur Verfügung stellen (http://letters1916. maynoothuniversity.ie/; Baillot 2016). Crowdsourcing und soziale Editionen werden bei der digitalen Aufbereitung von Briefmaterial vergleichsweise selten eingesetzt.

Autorzentrierte Briefaufbereitung nimmt in der Regel die Form einer Edition an (eine nennenswerte Ausnahme macht hier die archivarische und wissenschaftliche Erschließung von Chamissos Briefwechsel zusammen mit dem Rest des Nachlasses;

http://staatsbibliothek-berlin.de/diestaatsbibliothek/abteilungen/handschriften/nachlaesseautographen/projekte/abgeschlossene-projekte/nachlass-adelbert-von-chamisso/). Die ersten digital aufbereiteten Briefsammlungen sind mit einem digitalen Oberbau dem Printhabitus verpflichtet geblieben. Das ist der Fall bei Portalen, die Retrodigitalisierungen existierender Briefeditionen zusammenführen (bspw. Heinrich Heine: http://www.hhp.unitrier.de/ oder Friedrich Schlegel: http://www.blogs.uni-mainz.de/friedrich-schlegel/). Als die ersten, für die Weiterentwicklung von digitalen Briefeditionen konzeptionell entscheidenden Ressourcen gelten die Edition der Briefe Alfred Eschers

(https://www.briefedition.alfred-escher.ch/), die Carl-Maria-von-Weber Gesamtausgabe (die einen beträchtlichen Anteil an Briefmaterial enthält; http://webergesamtausgabe.de/de/Index) und die Edition der Briefe Vincent Van Goghs (http://vangoghletters.org/vg/).

Die Escher- und die Weber-Edition zeichnen sich insbesondere durch die systematische Umsetzung einer TEI-basierten Briefauszeichnung und die Verlinkung mit anderen digitalen Ressourcen über die Gemeinsame Normdatei aus (http://www.dnb.de/DE/Standardisierung/GND/gnd node.html; Stadler 2016). Dabei erheben sie Interoperabilität und Austauschmöglichkeiten zu einem grundsätzlichen Anspruch der editorischen Arbeit an und mit Briefen. Der entscheidende Beitrag der Van Gogh-Edition seinerseits ist auf der Ebene der Webanzeige anzusiedeln. Erstmalig ist dort die Verbindung von einfachem Zugang und fundierter Recherche in einer digitalen Briefedition gelungen. Die Gegenüberstellung der Scans und der Transkription, die zur Überprüfung des gelieferten Brieftextes sowie zu einer neuartigen Auseinandersetzung mit der Materialität des Briefs (sowie des in den Briefen erwähnten Werkmaterials) einlädt, setzt neue Standards hinsichtlich der an Briefeditionen gestellten Usability-Ansprüche (interessante, bis dato einmalige Analyse bei Boot 2011).

Ebenfalls nennenswert und autorzentriert ist die Edition der Korrespondenz August Wilhelm Schlegels, die einen diversifizierteren Einstieg in den Text ermöglicht als die traditionelle chronologische oder alphabetische Sortierung (http://www.august-wilhelmschlegel.de/briefedigital/). Diese Edition geht in der Verlinkung unterschiedlicher digitaler Ressourcen mit der Zusammenführung von Digitalisaten früherer Editionen und von für die digitale Edition eigens geleisteten, neuen Editionsarbeiten, die jeweils unterschiedlichen editorischen Ansätzen folgen, einen Schritt weiter.

Andere digitale Aufbereitungen von Briefmaterial gehen über die literaturwissenschaftlich traditionelle, autorzentrierte Herangehensweise hinaus und loten verstärkt das Potential 
aus, das mit der Verlinkung unterschiedlicher digitaler Ressourcearten zusammenhängt. So ist es möglich, Briefe mit anderen Briefen, mit in den Briefen erwähnten Werken oder sonstigen Materialien (vgl. van Goghs malerisches Werk), mit in den Briefen erwähnten Personen, komplex und in der Tiefe zu verbinden. Das Forschungsvorhaben Exilnetz (http://exilnetz33.de/de/) versammelt Briefmaterial, genauso wie es bei der Edition A.W. Schlegels der Fall ist, nicht ausschließlich bzw. vorrangig in Form eines edierten Textes, sondern über einen thematischen Thesaurus (Biehl et al. 2015). Die so beobachtbaren Netzwerke lassen zwar neuartige Analysen zu den Strukturen der deutschsprachigen Schriftsteller_innen im Exil im 2. Weltkrieg zu, lassen sich aber nicht auf andere Korpora eins-zu-eins übertragen oder leicht erweitern.

Auf ähnliche Formatschwierigkeiten bei der Verknüpfung von Briefmaterial stolpert ebenfalls das wesentlich umfangreicher angelegte, europäische Forschungsvorhaben „Reassembling the Republic of Letters", das im Rahmen einer COST-Action Archive, Bibliotheken und Forscher_innen verbindet, die sich mit gelehrten Briefen aus der Zeit zwischen 1500 und 1800 beschäftigen (http://www.republicofletters.net/). Besonders einschlägig in diesem Vorhaben ist die Beobachtung einer strukturellen Ähnlichkeit zwischen dem Kommunikationsnetzwerk der intellektuellen frühen Neuzeit und den neuen Medien. Die letzten zu verwenden, um die ersten abzubilden, ist ein in vielerlei Hinsicht reizvolles Unterfangen. Doch selbst diese groß angelegte Verbindung unterschiedlicher Instanzen eines spezifischen und strukturell feinpassenden Briefmaterials stößt an die Grenzen der dort vertretenen Methodenvielfalt: In Ermangelung eines gemeinsamen Standards ist es den vielen Archiven, Bibliotheken und Forschungsvorhaben nicht möglich, die Verlinkung ihres jeweiligen Briefmaterials zu operationalisieren.

Ein im Umfang viel kleinerer Versuch einer Verlinkung auf mehreren Ebenen (Briefmaterial mit den erwähnten Werken, mit weiterführenden biographischen Informationen zu verbinden; wissenschaftliche Aufbereitung mit archivarischer Erschließung zu kombinieren) ist im Rahmen der Briefedition Briefe und Texte aus dem intellektuellen Berlin um 1800 erfolgt, die ähnlich dem Exilnetz und der Republic of Letters einer Forschungsfrage entspringt und wo das edierte Material auf der Grundlage der vorhandenen Datenstruktur potentiell erheblich weiterwachsen kann (www.berliner-intellektuelle.eu). Der geringen Menge an tatsächlich edierten Texten ist die Entwicklungsarbeit der editorischen Richtlinien entgegenzusetzen, die eine interoperable Erweiterung möglich macht, ja vorsieht (Kodierung nach TEI-P5, Einbettung von Normdaten, Langzeit-Verfügbarmachung der XML-Quelldateien mit einer CC-BY-Lizenz). In diesem wie in vielen anderen Briefaufbereitungsprojekten befinden wir uns noch vor der eigentlichen wissenschaftlichen Reifezeit.

Die Kluft in der Vielfalt der bestehenden Ansätze bei der Digitalisierung von Briefmaterial lässt eher auf die Dauerhaftigkeit von Unternehmen schließen, die auf etablierte, interoperable und offene digitale Formate und Standards setzen. Wie sich dieses Forschungsfeld entwickeln und ob sich die (deutsche wie internationale) Briefforschung dem digitalen Potential gewachsen zeigen wird, ist jedoch noch offen. 


\section{Bibliographie}

Baillot, Anne (2016). „Was tun mit der Weisheit? Moderne Philologie im digitalen Zeitalter". Symphilologie. Formen der Kooperation in den Geisteswissenschaften, Hrsg. von Stefanie Stockhorst, Marcel Lepper und Vinzenz Hoppe. Göttingen. 261-279.

Baillot, Anne und Busch, Anna (2014). "'Berliner Intellektuelle um 1800' als Programm. Über Potential und Grenzen digitalen Edierens". Romantik digital, literaturkritik Sept. 2014 [http://www.literaturkritik.de/public/rezension.php?rez id=19678\&ausgabe=201409]

Beißwenger, Michael (2016). „Praktiken in der internetbasierten Kommunikation“. Sprachliche und kommunikative Praktiken. Jahrbuch 2015 des Instituts für Deutsche Sprache. Hrsg. v. Arnulf Deppermann, Helmuth Feilke und Angelika Linke.

Berlin/New York. 279-310.

Biehl, Theresia, Lorenz, Anne, Osierenski, Dirk (2015). „Exilnetz33. Ein Forschungsportal als Such- und Visualisierungsinstrument". Grenzen und Möglichkeiten der Digital Humanities (Zeitschrift für digitale Geisteswissenschaften, Sonderband 1.

[http://www.zfdg.de/sb001 011]

Bohnenkamp, Anne, Wiethölter, Waltraud (2008). Der Brief - Ereignis und Objekt. Frankfurt a. M.

Bohnenkamp, Anne, Wiethölter, Waltraud (2008). Der Brief - Ereignis und Objekt. Frankfurt

Boot, Peter (2011). "Reading Van Gogh Online?". Ariadne Issue 66

[http://www.ariadne.ac.uk/issue66/boot/]

Burghart, Marjorie (2017). "The TEI Critical Apparatus Toolbox: Empowering Textual Scholars through Display, Control, and Comparison Features ". Journal of the Text Encoding Initiative [Online], Issue 10 | 2016, Online since 26 December 2016, connection on 07 February 2017. URL: http://jtei.revues.org/1520 ; DOI : 10.4000/jtei.1520

Busch, Anna (2013). „,,Verwahre meine Briefe, Briefe sind Archive." Julius Eduard Hitzigs Leben und Briefe von Adelbert von Chamisso: Entstehungsgeschichte, Quellenlage, Programm, Rezeption“. Korrespondenzen und Transformationen. Neue Perspektiven auf Adelbert von Chamisso. Hrsg. von Marie-Theres Federhofer und Jutta Weber. Göttingen. 195-216.

Busch, Anna, Görbert, Johannes (2016). „,',Rezensiert und zurechtgeknetet. “ Chamissos Briefe von seiner Weltreise - Original und Edition in Gegenüberstellung". Phantastik und Skepsis. Adelbert von Chamissos Lebens- und Schreibwelten. Hrsg. von Roland Berbig, Walter Erhart, Monika Sproll und Jutta Weber. Göttingen. 111-144.

Gius, Evelyn, Jacke, Janina (2016). Zur Annotation narratologischer Kategorien der Zeit. Guidelines zur Nutzung des CATMA-Tagsets. Version 2. Hamburg. [http://heureclea.de/wpcontent/uploads/2016/11/guidelinesV2.pdf] 
ETH Bibliothek (2016). Best Practices Digitalisierung. Version 1.1. Zürich.

Neudecker, Clemens, Rehm, Georg (2016). „Digitale Kuratierungstechnologien für Bibliotheken“. Zeitschrift für Bibliothekskultur / Journal For Library Culture, 4/ 2. [http://0277.ch/ojs/index.php/cdrs 0277/article/view/158]

Schmid, Helmut (1994). "Probabilistic Part-of-Speech Tagging Using Decision Trees". Proceedings of International Conference on New Methods in Language Processing, Manchester. [http://www.cis.uni-muenchen.de/ schmid/tools/TreeTagger/data/treetagger1.pdf]

Schmid, Helmut (1995). "Improvements in Part-of-Speech Tagging with an Application to German". Proceedings of the ACL SIGDAT-Workshop. Dublin. [http://www.cis.unimuenchen.de/ schmid/tools/TreeTagger/data/tree-tagger2.pdf]

Schöch, Christof (2016). “Topic Modeling Genre: An Exploration of French Classical and Enlightenment Drama". Digital Humanities Quarterly.

http://doi.org/10.5281/zenodo.166356

Schreibman, Susan, Siemens, Ray, Unsworth, John (2004). A Companion to Digital Humanities. Oxford. [http://www.digitalhumanities.org/companion/]

Stadler, Peter (2016). „Normdateien in der Edition“. Editio, 26/ 1. 174-183.

[https://doi.org/10.1515/editio-2012-0013]

Stadler, Peter, Illetschko, Marcel, Seifert, Sabine (2016). "Towards a Model for Encoding Correspondence in the TEl: Developing and Implementing <correspDesc>". Journal of the Text Encoding Initiative [Online], Issue 9 | 2016, Online since 24 January 2017, connection on 07 February 2017. URL: http://jtei.revues.org/1433 ; DOI : 10.4000/jtei.1433

Strobel, Jochen (2012). „Digitale Briefeditionen. Eine Reflexion zu den Digital Humanities“. BIS Das Magazin der Bibliotheken in Sachsen, 5/ 03.

[http://www.qucosa.de/fileadmin/data/qucosa/documents/9673/BIS 3.12 SCREEN mediu $\underline{\text { m.pdfl }}$

TEI Consortium. 2013. TEI P5: Guidelines for Electronic Text Encoding and Interchange. Version 3.1.0. Last updated on 15th December 2016, revision d3f5e70 N.p.: TEl Consortium. http://www.tei-c.org/Vault/P5/2.5.0/doc/tei-p5-doc/en/html/. 